\title{
TWO CASES OF THROMBOSIS OF THE RETINAL VEIN, ONE SHOWING A HOLE, THE OTHER A STAR AT THE MACULA*
}

BY

\author{
F. Arnold Williamson, F.R.C.S. \\ I.ONDON
}

THE pathology of holes at the macula is a subject which, from time to time, has excited a good deal of attention and has been discussed at some length by the late Mr. George Coats in his paper in the R.L.O.H. Reports, Vol XVII, Part I, in which he summarises the various theories which have been put forward to account for the condition.

There is first the theory of Menteith Ogilvie, who accounts for the traumatic origin of a hole at the macula by stating that there are two factors concerned:-

(a) The retina is thinnest at the fovea and thicker around it than elsewhere.

(b) That waves of disturbance passing through the eye meet at the posterior pole and tear the fovea by " contre coup."

Fuchs attributes it in traumatic cases to a mild form of traumatic retinitis, the very mild toxic stimulus, reaching the retina from the anterior part of the eye, causing instead of exudation of pus, a slight serous exudate which ruptures the membrana limitans externa and so causes the appearance of a macular hole.

Coats supports the theory of oedema being the underlying cause of macular holes, stating that a hole has never been observed sooner than 60 hours after an injury, and, on the other hand, that opacity of the retina without a hole has been observed after injury in a case in which a hole subsequently developed. Also, that oedema of the retina and subsequent formation of a hole have been observed in cases where there had been no injury.

A year after the publication of this paper, however, Kipp and Alt, in the American Journal of Ophthalmology for August, 1908, reported the following case.

A boy was shot through the orbit, the bullet passing behind the eye, tearing the optic nerve and its sheaths, and entering the skull. He was seen on the day following, when the retina was noted to be whiteish and the vessels narrow, except for a vein passing from the macula to the disc. At the macula was a deep red round spot, one third P.D. Two days later the retina was more opaque and the blood vessels were fuller. Four days later the eye was excised and sections through the macula showed a large hole bounded by swollen retinal tissue. The choroidal vessels, especi-

* Read before the Section of Ophthalmology of the Royal Society of Medicine, on December 2, 1921. 
ally near the posterior pole, were very hyperaemic and distended, and evidences of oedema were visible everywhere. Arguing from this case, these authors consider that the retina at the macula can be torn by " contre coup," and state, moreover, that holes at the macula seem to be caused without exception by injuries to the orbit or eyeball, during which a contusion of the eyeball takes place.

The reason why I thought that the following cases would be of interest is, that especially in the first case, they show, in an almost

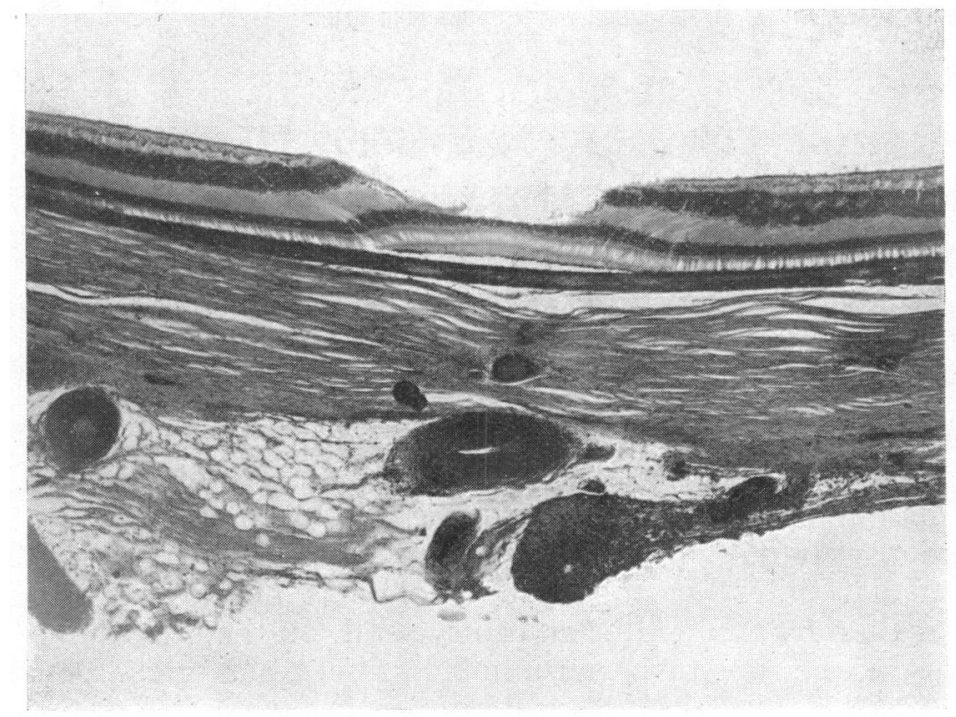

FiG. 1.

diagrammatic fashion, the dependence of the formation of a hole at the macula on the occurrence of sub-retinal oedema.

The salient points in the history are as follows :-

Age, 62 (female).

Sight began to fail 14 years ago, recommended to hospital and had teeth extracted.

Pains in the head 1919-1920. Severe pain August, 1920, and continuous more or less till excision in July, 1921, for absolute glaucoma.

She had a fall 30 years ago and blacked both eyes, but sight was not affected. Clinical examination showed a large corneal nebula with a few vessels. No perception of light. $T+1 . \mathrm{V}$. in other eye, with correction, $=6 / 9$. The eye was frozen in the usual way and bisected horizontally. On examination with the loupe there 
was seen a small but quite definite hole at the macula, on which parallax could be obtained. Microscopical examination of other parts of the excised eye showed the following changes :-

A slightly cellular exudate adherent to the posterior surface of the cornea in places.

Albumin in the angles of the anterior chamber.

Hyaline and faintly cellular exudate passing round the lens and causing an adhesion of the iris to it.

No cupping of the disc, and a thrombosed vein near its margin.

Serial celloidin sections were made of the disc and macula, and

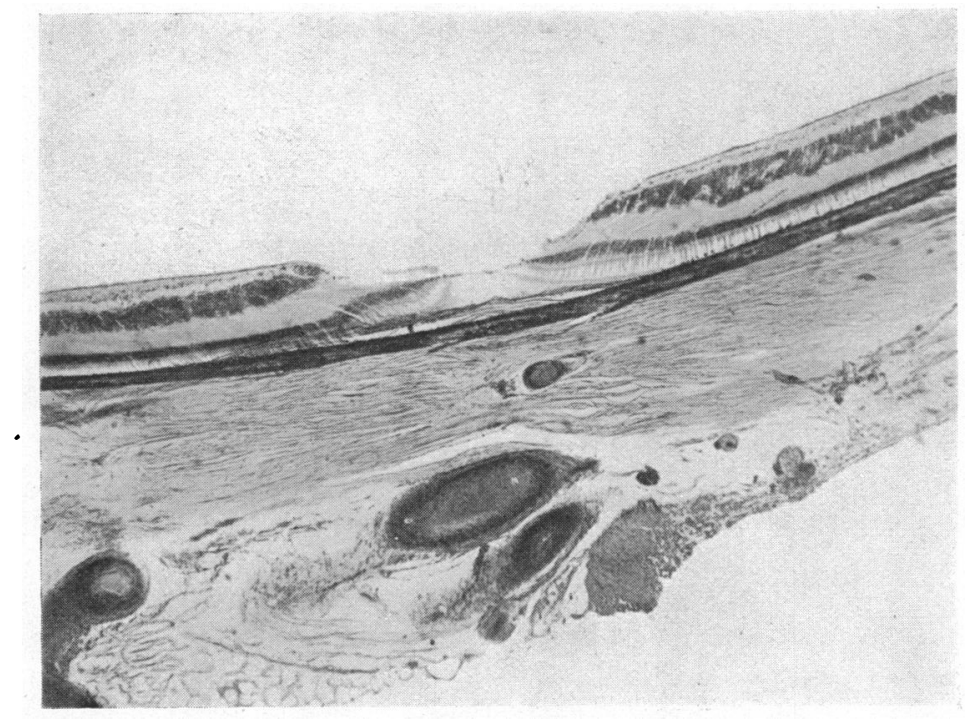

FIG. 2.

some of these are shown in the microphotographs accompanying this paper.

Remarks on sections :-

1. The macular region is shown here and one notices:-

(i) Absence of inner nuclear layer and inner reticular layer.

(ii) That the retinal pigment layer is separated from the inner layer of the choroid, a condition not present in other parts of the specimen.

(iii) That the rods are fairly well preserved, but that between them and the pigment layer is a thin layer of structureless material, presumably oedematous fluid, fixed by hardening reagents.

(iv) That there is a certain amount of oedema in the outer molecular layer of the peri-macular region.

2 . This is two sections nearer the fovea and one notices :- 
(i) Absence of the outer nuclear layer.

(ii) The layer of rods and cones is pushed forward by the accumulation of fluid behind.

(iii) That in this fluid there has developed a cyst, whose anterior wall is clearly formed only by the membrana limitans externa of the retina, i.e., the layer of rods over it has disappeared.

(iv) The area of separation of retinal pigment layer alluded to above is also present.

3. This section would appear to be at the fovea; it presents the

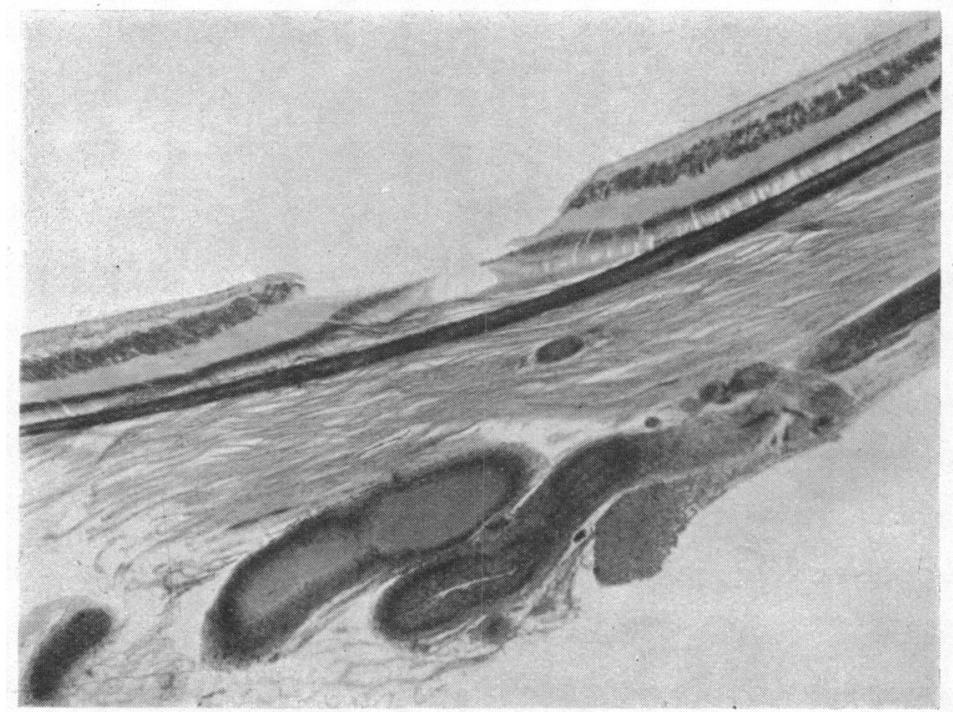

FIG. 3.

same characteristics as before, except that the anterior wall of the cyst is not present, thus causing the formation of a hole.

The feature to which. I would call special attention in these sections is that the cyst is formed, not in the retina but in the accumulation of oedematous material between the membrana limitans externa and the retinal pigment layer. And, secondly, that in consequence of the presence of this cyst, the outer layer of the retina would appear to undergo a degenerative process, thus allowing the formation of a hole.

Second case.- This patient had been blind in the R.E. for five years. Fourteen days before admission it became red and painful. $T+1$. No perception of light. Eye excised. Urine contained no sugar or albumin.

The eye was frozen and bisected. 
Pathological examination showed the following points :-

(i) The anterior chamber contained partly organized blood clot, which blocked its angles. Posterior synechiae were present and at the periphery the iris was atrophic and adherent to the posterior surface of the cornea. A faint cyclitic exudate was present round the lens, which showed cataractous changes. Round the cupped disc was a zone of haemorrhages and at the macula there was a faint star.

The changes at the macula bear a certain resemblance to those in the last case. The sections also showed :-

(i) The thrombosed vessel on the side of the disc.

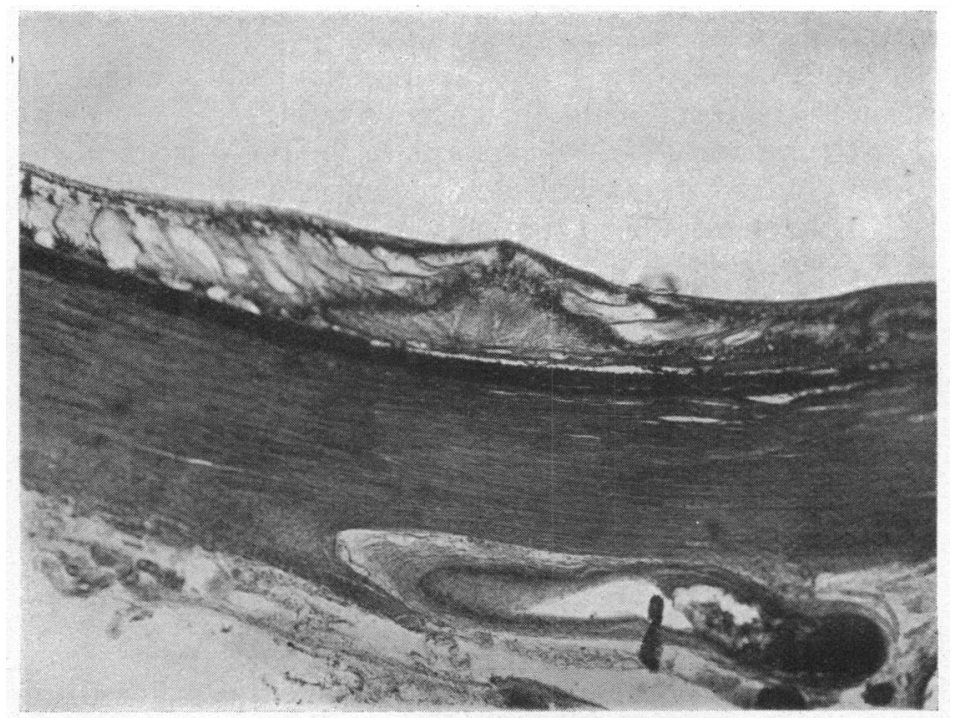

FIG. 4.

(ii) Slight cupping of the disc.

(iii) Some small haemorrhages in the inner layers of the retina.

(iv) Two-thirds of the way between disc and macula a small collection of coagulated fluid between the choroid and rod and cone layer of the retina. Owing, presumably to the degenerate state of the retina, one could not make out the retinal pigment laver in the same way as in the last case.

(v) At the macula (vide figure 4) a much larger collection of exudate, the retina here showing advanced cystic degeneration which beyond the macula can be seen to start in the outer reticular layer.

There is no macular hole present in this specimen, but one 
cannot help feeling that if the condition had lasted a little longer the retina might have degenerated still further and a hole would have formed, and possibly a cyst have developed in the sub-retinal exudate.

The first series of sections would seem to support the theory that a hole at the macula is due to sub-retinal oedema, and the second shows degeneration affecting an area of the retina separated from the choroid by a layer of oedema. Despite this, however, the whole pathology of the condition would not yet appear to be settled, since there are still two points to determine; first, the source of the oedema, and secondly, why it should be localized to the macular and sub-macular region.

Considering first of all the points in favour of the oedema arising from retinal vessels. They are as follows :-

(i) In both cases there is an obvious source of retinal venous congestion in the form of the thrombosed vein.

(ii) In the second series of sections in the peripheral part of the retina, "the degeneration was seen to start in the outer reticular layer. Now Leber showed that this part of the retina is supplied by the terminal branches of the retinal vessels, and would therefore be most sensitive to any changes in the retinal circulation.

(iii) Coats, in discussing the pathology of retinal disease with massive organization, states that the haemorrhages found in the early stages of this disease occur in the outer layers of the retina and break through into the sub-retinal space. If blood takes this path, it is possible for oedematous exudate to do so too.

Now turning to the points in favour of a choroidal origin for the oedema, we have the following:-

(i) The oedema is localized to the macular and sub-macular regions, while the macular portion of the retina is poorer in retinal capillaries than any other part, the fovea centralis being devoid of them and dependant on the chorio-capillaris for its blood supply.

(ii) Leber showed that the calibre of the choroidal capillaries is greatest at the macula and that the chorio-capillaris is thicker here than elsewhere. Arguing from the fact that this portion of the choroid is entirely responsible for the nourishment of the most functionally active part of the retina, one would expect the walls of the choroidai capillaries to be more permeable here than elsewhere so as to allow of the maximum transudation of nutrient materials. This being so, one would expect this part to be the first to suffer from the action of any toxin.

(iii) Hepburn, in the Royal London Ophthalmic Hospital Reports, Vol. XVIII, page 92, suggested the grouping of the posterior ciliary vessels into three separate divisions showing an absence of anastomosis with each other, one of these groups being destined solely for the macular region of the chorio-capillaris. 
This arrangement would account for the limitation of the oedema to the macular region.

(iv) In the first series of slides the oedema is most marked in the space between the rods and cones and the pigment epithelium. Now, in papilloedema the condition which Paton and Gordon Holmes have shown is due to obstruction to retinal venous and lymphatic return, oedema, when it occurs in the form of a macular fan is due to superficial vesicles which are formed by the raising of the membrana limitana interna from the nerve fibre layer.

(v) Finally, there is the point about the external molecular layer representing that part of the retina which would degenerate first in a case of deficient circulation through the retinal vessels. It is also the part likely to be first affected when cut off from the chorio-capillaris by a layer of oedema, since it is further away from the choroid than any of the other retinal layers which depend on this structure for their nutrition.

I would suggest, therefore, as a possible way out of the difficulty that in these two cases we have a toxic influence-shown by the presence of the irido-cyclitis-acting on the retinal veins causing thrombosis and acting on the delicate macular choroidal capillaries causing degeneration of their walls, increased permeability to fluid, and consequent oedema. This fluid then diffuses or osmoses through into the potential space between the rods and cones and the pigment epithelium, preventing the access of nutrient materials from the chorio-capillaris so that the superjacent retina degenerates and forms a hole.

In traumatic cases, it may be possible to reconcile the theory of Menteith Ogilvie with the oedema theory by regarding the macular chorio-capillaris as the meeting point of the radiating lines of force passing from front to back of the eye. Then, bearing in mind the delicacy of these capillaries, one can understand that if oedema is to be caused anywhere it should be here, and once the macular region of the retina has become separated from its source of nutrition it degenerates and forms a hole.

This seems to be going a long way round to explain the pathology of these cases, especially in the non-traumatic examples, but I can think of no other explanation which would account for the limitation of the oedema and for its occurence in the sub-retinal space.

In conclusion I am indebted to Mr. Mayou and Mr. Levy for their kind permission to use material derived from their cases in the preparation of the accompanying photographs, and to $\mathrm{Mr}$. Mayou for valuable advice as regards this paper. 